\title{
Author's response to "letter to the editor: lower vitamin D status may explain racial disparities in all-cause mortality among younger commercially insured women with incident metastatic breast cancer"
}

\author{
Christine Leopold ${ }^{1}$
}

Received: 14 July 2016/Accepted: 15 July 2016/Published online: 28 July 2016

(c) Springer Science+Business Media New York 2016

To the Editor,

Dr. Grant raises the possibility that lower vitamin D levels among black women caused the racial disparity in mortality which we detected in patients with metastatic breast cancer. Understanding the causes of breast cancer mortality disparities is crucial, but this was not the aim of our observational, claims-data-based study. Rather, we sought to assess, for the first time, whether disparities exist in mortality among younger insured women with metastatic breast cancer. Validly determining the causes of such disparities would require study designs and data sources other than those we employed. As we note in our discussion, the causes of such racial disparities are likely multifactorial, interconnected, and complex.
Further, Dr. Grant writes that “... the finding that the hazard ratio for survival is nonsignificantly elevated in the West and significantly decreased in the Northeast is likely due to the higher UVB doses in the West and the lower UVB doses in the Northeast." In fact, our finding was that metastatic breast cancer patients who live in the Northeast have the lowest mortality ( $9 \%$ vs. 15,13 , and $14 \%$ in the West, Midwest, and South, respectively), an adjusted hazard ratio of $0.73(0.56,0.95)$. As mentioned above, the reasons for such better outcomes among women in the Northeast are not revealed by our analysis, but are likely multifactorial.

With kind regards, Christine Leopold.
This rebuttal letter refers to the letter to the editor article available at doi:10.1007/s10549-016-3916-7.

Christine Leopold

christine_leopold1@hphc.org

1 Harvard Medical School and Harvard Pilgrim Health Care Institute, Boston, USA 